\title{
Pre-formed Cooper pairs and Bose-Einstein condensation in cuprate superconductors
}

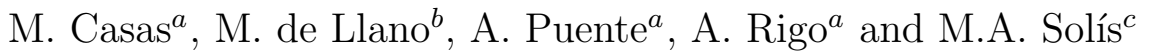 \\ ${ }^{a}$ Departament de Física, Universitat de les Illes Balears, \\ 07071 Palma de Mallorca, Spain \\ ${ }^{b}$ Instituto de Investigaciones en Materiales, Universidad Nacional Autónoma de México, \\ Apdo. Postal 70-360, 04510 México, DF, Mexico \\ ${ }^{c}$ Instituto de Física, Universidad Nacional Autónoma de México, \\ Apdo. Postal 20-364, 01000 México, DF, Mexico.
}

November 10, 2018

\begin{abstract}
A two-dimensional (2D) assembly of noninteracting, temperature-dependent, pre-formed Cooper pairs in chemical/thermal equilibrium with unpaired fermions is examined in a binary boson-fermion statistical model as the BoseEinstein condensation (BEC) singularity temperature $T_{c}$ is approached from above. Compared with BCS theory (which is not a BEC theory) substantially higher $T_{c}$ 's are obtained without any adjustable parameters, that fall roughly within the range of empirical $T_{c}$ 's for quasi-2D cuprate superconductors.
\end{abstract}

A possible interpretation of the "pseudogap" observed in some superconductors above $T_{c}$ is that it arises simultaneously with the formation of "pre-formed" Cooper pairs (CPs). We propose here that such objects emerge naturally as the nonzero-total (or, -center-of-mass) momentum (CMM) CPs that are entirely neglected in ordinary BCS theory.

Consider a 2D system of $\mathrm{N}$ fermions of mass $m$ confined in a square of area $L^{2}$ and interacting pairwise via the BCS model interaction $V_{\mathbf{k}, \mathbf{k}^{\prime}}=-V$ when $\mu(T)-\hbar \omega_{D}<$ $\epsilon_{k_{1}}\left(\equiv \hbar^{2} k_{1}^{2} / 2 m\right)$ and $\epsilon_{k_{2}}<\mu(T)+\hbar \omega_{D}$, and zero otherwise, where $\mathbf{k} \equiv \frac{1}{2}\left(\mathbf{k}_{1}-\mathbf{k}_{2}\right)$ is the relative wavevector of the two particles; $V_{\mathbf{k}, \mathbf{k}^{\prime}}$ the $2 \mathrm{D}$ double Fourier integral of the underlying non-local interaction $V\left(\mathbf{r}, \mathbf{r}^{\prime}\right)$ in the relative coordinate $\mathbf{r}=\mathbf{r}_{1}-\mathbf{r}_{2} ; \mu(T)$ the ideal Fermi gas (IFG) chemical potential which at $T=0$ becomes the Fermi energy $E_{F} \equiv \hbar^{2} k_{F}^{2} / 2 m$ with $k_{F}$ the Fermi wavenumber; $2 \hbar \omega_{D} \equiv \hbar^{2} k_{D}^{2} / m$ the energy width of the annulus centered around the Fermi circle and where the interaction is nonzero, with $\omega_{D}$ the Debye frequency. For $V>0$ this model interaction mimics the net effect of an attractive electron-phonon interaction overwhelming the interfermion Coulomb repulsions.

If $\hbar \mathbf{K}=\hbar\left(\mathbf{k}_{1}+\mathbf{k}_{2}\right)$ is the CMM of a CP, let $E_{K}$ be its total energy (besides the CP restmass energy). The original eigenvalue $\mathrm{CP}$ [1] equation for a pair of fermions at $T=0$ immersed in a background of $N-2$ inert, spectator fermions lying within a (sharp) Fermi circular perimeter of radius $k_{F}$, is then

$$
1=V \sum_{\mathbf{k}}^{\prime} \frac{\theta\left(k_{1}-k_{F}\right) \theta\left(k_{2}-k_{F}\right)}{2 \epsilon_{k}+\hbar^{2} K^{2} / 4 m-E_{K}},
$$

where $\theta(x)$ is the Heaviside unit step function, and the prime on the summation sign denotes the conditions $k_{1,2} \equiv\left|\frac{1}{2} \mathbf{K} \pm \mathbf{k}\right|<\left(k_{F}^{2}+\right.$ $\left.k_{D}^{2}\right)^{1 / 2}$ ensuring that our pair of fermions above the Fermi "surface" cease interacting beyond the annulus of energy width $2 \hbar \omega_{D}$, thereby restricting the summation over $\mathbf{k}$ for a given fixed K. Without these restrictions (1) would just be the Schrödinger equation in momentum space for the pair. Setting $E_{K} \equiv 2 E_{F}-\Delta_{K}$, the pair is bound if $\Delta_{K}>0$, and (11) becomes an eigenvalue equation for the (positive) pair binding energy $\Delta_{K}$. Our $\Delta_{K}$ and $\Delta_{0}$ should not be confused with the BCS energy gap $\Delta(T)$ at 
$T=0 . \quad$ Let $\lambda \equiv g\left(E_{F}\right) V \geq 0$ be the usual BCS dimensionless coupling constant. Here $g\left(E_{F}\right)$ is the electronic density-of-states (for each spin) at the Fermi surface in the normal (i.e., interactionless) state, which in 2D is $g(\epsilon)=L^{2} m / 2 \pi \hbar^{2} \equiv g$, a constant. The Cooper equation (11) for the unknown quantity $\Delta_{K}$ was analyzed in Ref. [2]. For zero CMM, $K=0$, it becomes a single elementary integral, with the familiar [1] solution $\Delta_{0}=$ $2 \hbar \omega_{D} /\left(e^{2 / \lambda}-1\right)$ valid for all coupling $\lambda$. For small $K$ one determines [2] for weak coupling, $\lambda \rightarrow 0$, that

$$
\Delta_{K} \underset{K \rightarrow 0}{\longrightarrow} \Delta_{0}-\frac{2}{\pi} \hbar v_{F} K+O\left(K^{2}\right)
$$

where $v_{F} \equiv \sqrt{2 E_{F} / m}$ is the Fermi velocity. This linear dispersion relation is the $2 \mathrm{D}$ ana$\log$ of the 3D result discussed as far back as 1964 in Ref. [3], p. 33 (see also, Ref. [4], p. 336, and 5]) but with the 2D coefficient $2 / \pi$ in (2) replaced by $1 / 2$. Though commonly confused with the Anderson-Bogoliubov-Higgs (ordinary) sound mode, the linear-dispersion result (2) corresponds to real, moving (preformed) CPs and is distinct from the zerocoupling $\mathrm{ABH}$ (indeed, IFG) phonons described by $\hbar v_{F} K / \sqrt{3}$. A general many-body formalism unambiguously exhibiting 6] this distinction involves solving the Bethe-Salpeter equation for Cooper pairing based not on the IFG, as above, but on the BCS ground-state in a Green's functions scheme allowing holes on a par with particles.

For $N_{B}$ ordinary bosons of mass $m_{B}$ and energy $\varepsilon_{K}=C_{s} K^{s}$ with $s>0$ and $C_{s}$ a constant, a temperature singularity appears at $T_{c} \neq 0$ for any [7] dimension $d>s$ in the number equation $N_{B}=\sum_{\mathbf{K}}\left[e^{\left(\varepsilon_{K}-\mu_{B}\right) / k_{B} T}-1\right]^{-1}$ at vanishing bosonic chemical potential $\mu_{B} \leq 0$ when the number of $\mathbf{K}=0$ bosons just ceases to be negligible upon cooling. It is given [8] by

$$
T_{c}=\frac{C_{s}}{k_{B}}\left[\frac{s \Gamma(d / 2)(2 \pi)^{d} n_{B}}{2 \pi^{d / 2} \Gamma(d / s) g_{d / s}(1)}\right]^{s / d}
$$

with $n_{B} \equiv N_{B} / L^{d}$ the boson particle density, and $g_{d / s}(z)$ the usual Bose integrals expressible as the series

$$
g_{\sigma}(z)=\sum_{l=1}^{\infty} \frac{z^{l}}{l^{\sigma}} \underset{z \rightarrow 1}{\longrightarrow} \zeta(\sigma),
$$

where $\zeta(\sigma)$ the Riemann zeta function of order $\sigma$. The last identification in (4) holds when $\sigma>$
1 for which $\zeta(\sigma)<\infty$, while the series $g_{\sigma}(1)$ diverges for $\sigma \leq 1$, thus giving $T_{c}=0$ for $d \leq 2$. For $s=2$ and $d=3$ one has $\zeta(3 / 2) \simeq 2.612$, and since $C_{2} \equiv \hbar^{2} / 2 m_{B}$ (3) then reduces to the familiar formula $T_{c} \simeq 3.31 \hbar^{2} n_{B}^{2 / 3} / m_{B} k_{B}$ of "ordinary" Bose-Einstein condensation (BEC). On the other hand, for either particle or hole bosons with (positive) excitation energy $\varepsilon_{K} \equiv$ $\Delta_{0}-\Delta_{K}$ given asymptotically by the linear term in (2) for all $K$, we have $C_{1} \equiv a(d) \hbar v_{F}$ where [9] $a(d)=1,2 / \pi$ and $1 / 2$ for $d=1,2$ and 3 , respectively. Now $T_{c}$ is nonzero for all $d>1$ - which is precisely the dimensionality range of all known superconductors including the quasi-1D organo-metallic (Bechgaard) salts [10.

The number of bosons in the boson-fermion mixture in chemical/thermal equilibrium turn out 111 to be temperature-dependent, and it is in conserving the fermion number that the singularity arises. As in the case of the pure boson gas (3), a linear rather than a quadratic dispersion relation is needed to obtain BEC in exactly 2D. All this emerges in a statistical model for the ideal binary gas mixture of bosons (the CPs) and unpaired (both pairable and unpairable) fermions in chemical equilibrium [12]. Thermal pair-breaking of the bosons into unpaired pairable fermions is explicitly allowed. At any $T$ the total number of fermions in $2 \mathrm{D}$ is $N=L^{2} k_{F}^{2} / 2 \pi=N_{1}+N_{2}$, and is just the number of non-interacting (i.e., unpairable) fermions $N_{1}$ plus the number of pairable ones $N_{2}$. The unpairable fermions obey the usual Fermi-Dirac (FD) distribution with the IFG chemical potential $\mu$. On the other hand, the $N_{2}$ pairable fermions are simply those in the interaction shell of energy width $2 \hbar \omega_{D}$ so that

$$
N_{2}=2 \int_{\mu-\hbar \omega_{D}}^{\mu+\hbar \omega_{D}} d \epsilon \frac{g(\epsilon)}{e^{\beta(\epsilon-\mu)}+1}=2 g \hbar \omega_{D},
$$

where $\beta \equiv\left(k_{B} T\right)^{-1}$, since a constant $g(\epsilon)$ renders the remaining integral exact. At any interfermionic coupling and temperature these fermions form an ideal mixture of pairable but unpaired fermions plus CPs that are created near the single-fermion energy $\mu(T)$, with binding energy $\Delta_{K}(T) \geq 0$ and total energy

$$
E_{K}(T) \equiv 2 \mu(T)-\Delta_{K}(T) .
$$

This generalizes the $T=0$ definition $E_{K} \equiv$ $2 E_{F}-\Delta_{K}$ given below (1).

The Helmholtz free energy $F \equiv E-$ $T S$, where $E$ is the internal energy and $S$ 
the entropy, for this binary gas "composite boson/pairable-but-unpaired-fermion system" at $T \leq T_{c}$ is then readily constructed 11 in terms of: a) $n_{2}(\epsilon)$, the average number of unpaired but pairable fermions with energy $\epsilon$; b) $N_{B, 0}(T)$, the number of (bosonic) CPs with zero CMM at temperature $T$; and c) $N_{B, K}(T)$, that number of excited pre-formed CPs (i.e., with arbitrary nonzero CMM $K$ ) and a cutoff $K_{0}$ physically defined [2] by $\Delta_{K_{0}} \equiv 0$ denoting the value of $K$ beyond which a CP breaks up. The free energy $F_{2}$ of the pairable fermions is to be minimized subject to the constraint that $N_{2}$ is conserved. If $N_{20}(T)$ is the number of pairable but unpaired fermions, the relevant number equation for the pairable (i.e., active) fermions is then

$$
\begin{aligned}
N_{2} & =N_{20}(T)+2\left[N_{B, 0}(T)+N_{B, 0<K<K_{0}}(T)\right] \\
& \equiv N_{20}(T)+2 N_{B}(T),
\end{aligned}
$$

where $N_{B, 0<K<K_{0}}(T)$ denotes the total number of "excited" bosonic pairs (namely with CMM such that $\left.0<K<K_{0}\right)$, i.e., $N_{B, 0<K<K_{0}}(T) \equiv$ $\sum_{0<K<K_{0}} N_{B, K}(T)$. At $T=0$ two distinct coupling regimes emerge: a) for $\Delta_{0}<2 \hbar \omega_{D}$ or for $\lambda \leq 2 / \ln 2 \simeq 2.89$, we have that $N_{20}(0)=$ $g\left(2 \hbar \omega_{D}-\Delta_{0}\right)$; while b) for $\Delta_{0}>2 \hbar \omega_{D}$ (or $\lambda \geq 2.89), N_{20}(0)$ is identically zero. Hence, the number of bosons $N_{B}(0)$ at $T=0$ from (7) is just $N_{B}(0)=\frac{1}{2}\left[N_{2}-N_{20}(0)\right]$. Using (5) for $\mathrm{N}_{2}$ the fractional number of pairable fermions that are actually paired at $T=0$, namely $2 N_{B}(0) / N_{2}=1-N_{20}(0) / N_{2}$, becomes simply $\Delta_{0} / 2 \hbar \omega_{D}=\left(e^{2 / \lambda}-1\right)^{-1} \longrightarrow e^{-2 / \lambda}$ as $\lambda \rightarrow 0$, for $\lambda \leq 2 / \ln 2 \simeq 2.89$, and unity for $\lambda \geq 2 / \ln 2$. As $N_{B}(0)=\frac{1}{2} g \Delta_{0}$ for $\lambda \leq 2.89$, only those fermions in an energy shell of width $\frac{1}{2} \Delta_{0}$ around the Fermi surface actually pair at $T=0$, while for $\lambda \geq 2.89$ all pairable fermions pair up since then $N_{B}(0)=g \hbar \omega_{D} \equiv \frac{1}{2} N_{2}$. For $T>0, \quad 2 N_{B}(T) / N_{2}=1-N_{20}(T) / N_{2} d e-$ creases with $T$, provided one knows $\Delta_{0}(T)$ for any $T \geq 0$ and ascertains that it decreases. For $T>0$, the $\theta\left(k_{1}-k_{F}\right) \equiv \theta\left(\epsilon_{k_{1}}-E_{F}\right)$ in (11) becomes $1-n\left(\xi_{k_{1}}\right)$, where $n\left(\xi_{k_{1}}\right) \equiv\left(e^{\beta \xi_{k_{1}}}+1\right)^{-1}$ is the FD distribution with $\xi_{k_{1}} \equiv \epsilon_{k_{1}}-\mu(T)$, with the IFG chemical potential $\mu(T)$ in $2 \mathrm{D}$ given exactly by $\mu(T)=\beta^{-1} \ln \left(e^{\beta E_{F}}-1\right) \longrightarrow E_{F}$ as $T \rightarrow 0$. Similarly for $\theta\left(k_{2}-k_{F}\right)$. Since $k_{1}=k_{2}$ implies that $\xi_{k_{1}}=\xi_{k_{2}}$, (11) then leads to a simple generalization to nonzero $T$ of the $K=0$ $\mathrm{CP}$ equation,

$$
1=\lambda \int_{0}^{\hbar \omega_{D}} d \xi\left(e^{-\beta \xi}+1\right)^{-2}\left[2 \xi+\Delta_{0}(T)\right]^{-1} .
$$

Numerical solution shows $\Delta_{0}(T)$ to indeed be monotonic-decreasing in $T$ for any fixed $\lambda$ and $\hbar \omega_{D}$. Further, the solution of $\Delta_{0}\left(T^{*}\right)=0$ is, by inspection, $T^{*}=\infty$; this infinite "de-pairing" temperature is unrealistic and undoubtedly an artifact of the simplest version (1) of Cooper pairing used here as a starting point; see, however, Ref. [6].

Modeling our system as a pure boson gas of CPs (i.e., neglecting the background unpaired fermions) but with a temperature-dependent number density $n_{B}(T)$ converts the explicit $T_{c^{-}}$ formula (3) into an implicit one. For $s=1$ and $d=2$ it becomes, since $g_{2}(1) \equiv \zeta(2)=\pi^{2} / 6$,

$$
T_{c}=\frac{4 \sqrt{3}}{\pi^{3 / 2}} \frac{\hbar v_{F}}{k_{B}} \sqrt{n_{B}\left(T_{c}\right)} .
$$

This requires $n_{B}(T) \equiv N_{B}(T) / L^{2}$ which in turn requires $\Delta_{0}(T)$ as determined from (8), and follows from the expression $2 N_{B}(T) / N_{2}=$ $1-N_{20}(T) / N_{2}$. Solving this self-consistently with (7) for $\lambda=1 / 2$ gives the remarkably constant value $T_{c} / T_{F} \simeq 0.004$, where $T_{F} \equiv$ $E_{F} / k_{B}$, over the entire range of $\nu \equiv \hbar \omega_{D} / E_{F}$ values $0.03-0.07$ typical of cuprate superconductors. On the other hand, the BCS formula $T_{c}^{B C S} \simeq 1.13 \Theta_{D} e^{-1 / \lambda}$ with $\lambda=1 / 2$ gives $T_{c} / T_{F}=0.005$ to 0.011 over the same range of $\nu$ values. Obviously, both sets of predictions are too small compared with the empirical cuprate range $T_{c} / T_{F} \simeq 0.03-0.09$ [13].

The exact $T_{c}$ without neglecting the background unpaired fermions requires the exact $\mathrm{CP}$ excitation energy dispersion relation $\varepsilon_{K}(T) \equiv \Delta_{0}(T)-\Delta_{K}(T)$ which is neither precisely linear in $K$ nor independent of $T$. To determine $\Delta_{K}(T)$ we need a working equation that generalizes Ref. [2 for $T>0$ via the new CP eigenvalue equation (8). At $T=T_{c}$ both $N_{B, 0}\left(T_{c}\right) \simeq 0$ and $\mu_{B}\left(T_{c}\right) \simeq 0$ so that one gets 111 the implicit $T_{c}$-equation for the binary gas mixture

$$
\begin{aligned}
1= & \frac{\tilde{T}_{c}}{\nu} \ln \left[\frac{1+e^{-\left\{\tilde{\Delta}_{0}\left(\tilde{T}_{c}\right) / 2-\nu\right\} / \tilde{T}_{c}}}{1+e^{-\left\{\tilde{\Delta}_{0}\left(\tilde{T}_{c}\right) / 2+\nu\right\} / \tilde{T}_{c}}}\right]+\frac{8(1+\nu)}{\nu} \\
& \times \int_{0}^{\kappa_{0}\left(\tilde{T}_{c}\right)} d \kappa \frac{\kappa}{e^{\left[\tilde{\Delta}_{0}\left(\tilde{T}_{c}\right)-\tilde{\Delta}_{\kappa}\left(\tilde{T}_{c}\right)\right] / \tilde{T}_{c}}-1}, \quad(10)
\end{aligned}
$$

where quantities with tildes are in units of $E_{F}$ or $T_{F} ; \kappa \equiv K / 2\left(k_{F}^{2}+k_{D}^{2}\right)^{\frac{1}{2}}$ with $k_{D}$ defined through $\hbar \omega_{D} \equiv \hbar^{2} k_{D}^{2} / 2 m$; and $\nu \equiv \Theta_{D} / T_{F}$. To obtain $T_{c}$ from the finite- $T$ dispersion relation one must numerically solve four equations selfconsistently for each $\lambda$ and $\nu$, namely (10) in 
conjunction with (8) for $\tilde{\Delta}_{0}(\tilde{T})$, and Eq. (35) of Ref. [2] for both $\tilde{\Delta}_{\kappa}(\tilde{T})$ and the breakup value $\kappa_{0}\left(T_{c}\right)$. For $\lambda=1 / 2$ and the range of $\nu$ values $0.03-0.07$ typical of cuprates, the resulting $T_{c} / T_{F}$ falls within the aforementioned empirical range $0.03-0.09$ 13. For cuprates $d \simeq 2.03$ has been suggested |14 as more realistic since it reflects inter-CuO-layer couplings, but our results in that case would be very close to those for $d=2$ since, e.g., from (3) $T_{c}$ for $s=1$ (but not for 2) varies little with $d$ around $d=2$. In fact, if $m_{B \perp}$ and $m_{B}$ are the boson masses perpendicular and parallel, respectively, to the cuprate planes, an "anisotropy ratio" $m_{B} / m_{B \perp}$ varied from 0 to 1 allows "tuning" $d$ continuously from 2 to 3 .

Other boson-fermion models [15 |16] 17 |18 19 have been introduced, some even addressing [17] 18] $d$-wave interaction effects as opposed to the pure $s$-wave considered here, and some also focus [18] [19] on the pseudogap. But calculating cuprate $T_{c}$ values in quasi-2D without adjustable parameters has not been attempted, and indeed $T_{c} \equiv 0$ is predicted in exactly $2 \mathrm{D}$.

In summary, a simple statistical model treating pre-formed CPs with both zero and nonzero CMM as non-interacting bosons in chemical/thermal equilibrium with unpaired fermions is proposed that gives rise to a boson number that is strongly coupling- and temperature-dependent. Since the CP dispersion relation is approximately linear for nonzero CMM, it exhibits a BEC of zero-CMM pairs at precisely $2 \mathrm{D}$. In contrast to both BCS theory - which is not 20] a BEC theory - and simpler BE models excluding either the breakable character of the pre-formed CPs or the presence of unpaired fermions, exact $T_{c}$ 's for the boson-fermion binary mixture based upon the exact $\mathrm{CP}$ dispersion relation are found in rough agreement with empirical cuprate values with no adjustable parameters.

M.C., A.P. and A.R. are grateful for partial support from grant PB98-0124, and M.deLl. from grants PB92-1083 and SAB950312, both by DGICYT (Spain), and from PAPIIT IN102198-9 as well as CONACyT 27828E (both Mexico). M.deLl. thanks P.W. Anderson, D.M. Eagles, R. Escudero, S. Fujita, M. Fortes, K. Levin, O. Rojo, A.A. Valladares for discussions, and V.V. Tolmachev for correspondence as well.

\section{References}

[1] L.N. Cooper, Phys. Rev. 104 (1956) 1189.

[2] M. Casas et al., Physica C 295 (1998) 93.

[3] J.R. Schrieffer, Theory of Superconductivity, (Benjamin, NY, 1964).

[4] A.L. Fetter et al., Quantum Theory of Many-Particle Systems, (McGraw-Hill, NY, 1971).

[5] S.K. Adhikari et al., Phys. Rev. B 62 (2000) 8671 and Physica C 351 (2001) 341.

[6] M. Fortes et al., Physica C 364-365 (2001) 95.

[7] J.D. Gunton et al., Phys. Rev. 166 (1968) 152; R.M. Ziff et al., Phys. Rep. 32 (1977) 169 .

[8] M. Casas et al., Phys. Lett. A 245 (1998) 55.

[9] S. Fujita et al., Quantum Statistical Theory of Superconductivity (Plenum, NY, 1996).

[10] D. Jérome, Science 252 (1991) 1509; J. M. Williams et al., Science 252 (1991) 1501.

[11] M. Casas et al., Physica A 295 (2001) 146.

[12] M.R. Schafroth, Phys. Rev. 96 (1954) 1442; M.R. Schafroth et al., Helv. Phys. Acta 30 (1957) 93.

[13] C.P. Poole, Jr. et al., Superconductivity (Academic, NY, 1995) p. 599.

[14] X.-G. Wen et al., Phys. Rev. B 37 (1988) 595.

[15] J. Ranninger et al., Physica B 135 (1985) 468.

[16] R. Friedberg and T.D. Lee, Phys. Rev. B 40 (1989) 6745.

[17] V. B. Geshkenbein et al., Phys. Rev. B 55 (1997) 3173.

[18] Q. Chen et al., Phys. Rev. B 59 (1999) 7083.

[19] T. Domanski et al., Phys. Rev. B 63, (2001) 134505.

[20] J. Bardeen, Physics Today (Jan. 1963) p. 25. 